\title{
CONHECIMENTO SOBRE ATIVIDADE FÍSICA E SAÚDE DOS PROFISSIONAIS DE ACADEMIAS DE PELOTAS, RS, BR
}

KNOWLEDGE ABOUT PHYSICAL ACTIVITY AND HEALTH BY GYM INSTRUCTORS OF PELOTAS, RS, BR

CONOCIMIENTO SOBRE ACTIVIDAD FÍSICA Y SALUD DE LOS PROFESIONALES DE GIMNASIOS

EN PELOTAS, RS, BR

Mateus Salerno'

(Educador Físico)

Airton José Rombaldi ${ }^{1}$

(Educador Físico)

Felipe Fossati Reichert ${ }^{1}$

(Educador Físico)

Marcelo Cozzensa da Silva'

(Educador Físico)

1. Universidade Federal de Pelotas, Pelotas, RS, Brasil.

\section{Correspondência:}

Universidade Federal de Pelotas, PPG em Educação Física, Rua Luis de Camões, 625, 96055-630,

Pelotas, RS, Brasil.

mateus_salerno@yahoo.com.br

\section{RESUMO}

Introdução: As academias de ginástica são locais para a prática de atividade física orientada. Apesar disso, muitos profissionais que nela atuam não apresentam formação universitária. Objetivo: Verificar o nível de conhecimento sobre atividade física e saúde e fatores associados dos profissionais que trabalham nas academias de ginástica na cidade de Pelotas, RS, Brasil. Métodos: Foi realizado um estudo censitário, com 497 profissionais que ministravam aulas nas academias. A coleta de dados, realizada por meio de questionário, ocorreu de novembro de 2011 a maio de 2012. O nível de conhecimento foi analisado por um escore gerado a partir de indicadores elaborados para o estudo. Resultados: A maioria dos indivíduos da amostra era do sexo masculino, com idade média de 29,7 $\pm 8,5$ anos, solteiros, com cor de pele branca e ensino superior completo. A média de acertos nas questões relativas ao conhecimento sobre atividade física e saúde foi de 10,6 $\pm 3,4$ pontos de um total de 20 . Ser do sexo masculino, faixa etária jovem e cor da pele branca estiveram associados a maiores escores de conhecimento. Além disso, indivíduos com maior renda e escolaridade obtiveram, em média, 2,1 e 3,8 pontos, respectivamente, a mais no escore de conhecimento quando comparados com indivíduos de menor renda e escolaridade. Conclusão: O nível de conhecimento sobre atividade física e saúde mostrou-se baixo entre os profissionais de academia, principalmente entre aqueles sem formação em educação física. É notória a necessidade de estratégias para melhorar o conhecimento desse grupo de profissionais.

Palavras-chave: docentes, educação física e treinamento, atividade motora, censos.

\section{ABSTRACT}

Introduction: Gyms are places for the practice of oriented physical activity. Despite that, many professionals working therein have no college education. Objective: To verify the level of knowledge about physical activity and health and associated factors of fitness center instructors in the city of Pelotas, RS, Brazil. Methods: We carried out a census study with 497 professionals who ministered classes at fitness centers in the city. Data was collected by questionnaire and took place from November 2011 to May 2012. The level of knowledge was analyzed using a score generated as from indicators elaborated for the study. Results: The majority of the individuals in the sample were male, mean age $29.7 \pm 8.5$ years old, single, White, and had college degree. The mean of right answers related to knowledge about physical activity and health was $10.6 \pm 3.4$ points out of a total of 20. Being male, young and White were associated with higher knowledge scores. Moreover, individuals with higher income and schooling obtained, in average, 2.1 and 3.8 points, respectively, more in the knowledge score when compared to those with lower income and schooling. Conclusion: The level of knowledge about physical activity and health showed to be low among gym's professionals and particularly among those without college degree in physical education. It is clear the need for strategies to improve the knowledge of this group of professionals.

Keywords: teachers, physical education and training, motor activity, censuses.

\section{RESUMEN}

Introducción: Los gimnasios son locales para práctica de actividad fisica guiada. Sin embargo, muchos profesionales no tienen educación universitaria. Objetivo: Determinar el nivel de conocimiento sobre la actividad física y la salud y los factores asociados de los profesionales que trabajan en los gimnasios de la ciudad de Pelotas, RS, Brasil. Métodos: Se realizó un estudio de censo con 497 profesionales que imparten clases en las academias de gimnasia. La recolección de datos, realizada a través de un cuestionario, se llevó a cabo entre noviembre de 2011 a mayo de 2012. El nivel de conocimiento fue analizado por un marcador generado a partir de los indicadores desarrollados para el estudio. Resultados: La mayoría de los individuos de la muestra eran hombres, con edad promedio de 29,7 \pm 8,5 años, solteros, de piel blanca y habían completado la educación superior. El promedio de éxito en los temas relacionados con el conocimiento de la actividad física y la salud fue de 10,6 23,4 puntos de un total de 20. Ser masculino, joven y color de la piel blanca fue asociada con las puntuaciones más altas de conocimiento. Además, las personas con ingresos más altos y educación superior 
tenían, en media, las puntuaciones de conocimiento 2,1 y 3,8 puntos, respectivamente, más altos en comparación con el promedio de las personas de bajos ingresos y educación. Conclusión: El nivel de conocimiento sobre la actividad física y la salud ha demostrado ser bajo entre los profesionales de la salud, especialmente entre aquellos que no tienen formación en educación física. Estos hallazgos subrayan la necesidad de estrategias para mejorar el conocimiento de este grupo de profesionales.

Palabras clave: docentes, educación y entrenamiento físico, actividad motora, censos.

\section{INTRODUÇÃO}

O processo de urbanização, industrialização e desenvolvimento tecnológico ocorrido nas últimas décadas tem acarretado modificações negativas no estilo de vida da população, o que contribuiu para o aumento das doenças e agravos não transmissíveis (DANTs) ${ }^{1}$. Um desses agravos, o sedentarismo, é conhecido atualmente como um problema de saúde mundial. Ele está associado a doenças cardiovasculares, alguns tipos de câncer, diabetes, entre outros problemas que acometem a saúde da população em geral².

A prática regular de atividade física (AF) possibilita diversos benefícios físicos e psicológicos à saúde, independentemente de fatores como idade, sexo, nível cultural e econômico, presença de incapacidades, entre outros ${ }^{3}$. Nos países desenvolvidos, os níveis de AF no lazer parecem aumentar, tornando a população mais ativa ${ }^{4,5}$. Entretanto, no Brasil, uma pesquisa realizada em todas as capitais incluindo o Distrito Federal relatou prevalências de prática insuficiente no lazer no Brasil de aproximadamente $70 \%{ }^{6}$.

A falta de espaços públicos, em grande parte dos municípios brasileiros, destinado à prática de AF, com infraestrutura física e pessoal e segurança adequada, têm feito com que muitos indivíduos procurem centros especializados na orientação desse tipo de atividade. As academias de ginástica, centros de condicionamento físico que oportunizam o ambiente e orientação profissional para a prática de programas de exercícios físicos ${ }^{7}$, apresentam potencial para influenciar o estilo de vida da população e possibilitar melhorias em seu bem-estar geral ${ }^{8}$. Nesse sentido, a existência de profissionais nesses locais com conhecimento técnico-científico para orientação qualificada sobre AF é de fundamental importância para a saúde e prescrição de atividades planejadas aos frequentadores. Entretanto, a profissão de educador físico é relativamente nova e, portanto, a existência no mercado de trabalho de indivíduos não graduados ainda persiste.

O conhecimento técnico dos profissionais de educação física em relação às DANTs ainda deve ser aprimorado ${ }^{9}$. Devido ao crescimento do número de academias, bem como de profissionais de educação física e leigos neste mercado de trabalho, o objetivo do presente estudo foi verificar o nível de conhecimento sobre AF e saúde e fatores associados dos profissionais graduados, estudantes de graduação, provisionados, não graduados e não estudantes de Educação Física que atuavam como orientadores de AF nas academias de ginástica da zona urbana da cidade de Pelotas, RS, Brasil.

\section{MÉTODOS}

Foi realizado em Pelotas, cidade localizada no sul do estado do Rio Grande do Sul, Brasil, com população aproximada de 328 mil habitantes, sendo cerca de $90 \%$ residentes na área urbana, um censo entre os profissionais atuantes em academias. Tratou-se de estudo epidemiológico observacional de corte transversal.

O protocolo do estudo foi submetido ao Comitê de Ética em Pesquisa da Escola Superior de Educação Física da Universidade Federal de Pelotas, RS, Brasil e aprovado sob o no 021/2011. Todos os participantes do estudo assinaram o Termo de Consentimento Livre e Esclarecido.

A logística do trabalho para encontrar as academias de ginástica, bem como o processo da coleta dos dados e a definição da população estudada está descrita no estudo de Hartwig et al. ${ }^{10}$, que visou estudar as condições de saúde dos profissionais que trabalhavam nestas academias.

O instrumento utilizado para a coleta de dados foi um questionário elaborado contendo questões demográficas (sexo: masculino/ feminino; idade: anos completos; cor da pele: branco/não branco; estado civil: casado-vive com companheiro/solteiro), econômica (renda bruta), social (escolaridade: ensino fundamental incompleto/ensino fundamental completo/ensino médio incompleto/ensino médio completo/ensino superior incompleto/ensino superior completo), nutricional (índice de massa corporal - IMC: calculado através do peso e altura autorreferidos e classificado em eutrófico - $\leq 24,9 \mathrm{~kg} / \mathrm{m}^{2}$, sobrepeso - entre 25,0 e $29,9 \mathrm{~kg} / \mathrm{m}^{2}$, obesidade $-\geq 30,0 \mathrm{~kg} / \mathrm{m}^{2}$ ) e de conhecimento sobre AF e saúde. A variável nível econômico foi definida a partir da renda bruta total dos entrevistados, e para as análises, foi dividida em quartis.

As questões de conhecimento sobre AF e saúde tinham por objetivo verificar os benefícios da AF para a saúde, prevenção de DANTs, finalidades da AF e efeitos crônicos da mesma no organismo humano, totalizando 20 questões fechadas que eram lidas aos entrevistados. As questões foram elaboradas baseando-se em questionários de estudos já existentes ${ }^{11,12,13}$, além de questões formuladas pelos próprios pesquisadores. Com base nos dados do questionário, gerou-se um escore de conhecimento, o qual foi obtido por meio das respostas corretas e a pontuação total variou entre zero e 20 pontos, sendo que cada questão possuía o valor de um ponto.

Durante o trabalho de campo, para fins de controle de qualidade, uma amostra aleatória de 10\% dos profissionais das academias foi novamente entrevistada pelos coordenadores do estudo, para aplicação de um questionário contendo perguntas-chave para a verificação de possíveis erros e fraudes.

A estruturação do banco de dados foi realizada no programa EpiData 3.1 (The EpiData Association, Odense, Denmark). Cada questionário foi duplamente digitado, permitindo comparar os bancos de dados e corrigir possíveis erros de digitação. Para análise dos dados, utilizou-se o software estatístico STATA 10.0 (StataCorp, Texas, USA). Primeiramente, realizou-se uma análise descritiva das variáveis em estudo (cálculo de médias e desvio-padrão [DP] para as variáveis contínuas e cálculo de proporções para as variáveis categóricas). Para verificação da normalidade de distribuição de escores utilizou-se o teste de Shapiro-Wilk. Posteriormente, associações foram verificadas, através do teste $t$ de Student e ANOVA One-Way. A análise multivariável foi realizada através de regressão linear múltipla, tendo como desfecho o nível de conhecimento na forma contínua. Foi analisada a influência das variáveis sexo, idade, cor da pele, estado civil, escolaridade, nível econômico e IMC sobre o nível de conhecimento. Foi adotado $\mathrm{p}<0,05$ como valor de significância. 


\section{RESULTADOS}

Foram encontradas 170 academias e 569 profissionais atuantes, dos quais foram entrevistados 519, entre proprietários e profissionais. Dos proprietários ( $\mathrm{n}=183$ ), 22 não atuavam ministrando aulas e, portanto, foram excluídos das análises. A porcentagem final de perdas foi de $5,1 \%$ e de recusas de 3,9\%.

A média de idade dos 497 profissionais atuantes em academias entrevistados foi de 29,7 anos ( $D P=8,5$, amplitude=17 a 65 anos), sendo que $57,9 \%$ eram do sexo masculino, 29,4\% casados/viviam com companheiro e $87,1 \%$ de cor da pele branca. Apenas 53,3\% dos profissionais possuía graduação em Educação Física e pouco menos da metade dos professores de Educação Física formou-se nos últimos 10 anos. Cerca de 12,1\% possuía outra graduação que não a de Educação Física e, aproximadamente, 10\% possuía apenas o ensino médio completo ou menos. A média de IMC entre os profissionais atuantes nesses estabelecimentos foi de $24,7 \mathrm{~kg} / \mathrm{m}^{2}$ ( $\left.\mathrm{DP}=3,4\right)$, sendo que $42,9 \%$ foram classificados nas categorias de sobrepeso/obesidade (tabela 1).

A média de acertos nas questões relativas ao conhecimento sobre atividade física e saúde foi de 10,6 $( \pm 3,4)$ pontos de um total de 20. Na análise bivariada, o conhecimento apresentou uma tendência direta de aumento à medida que aumentou a escolaridade e o nível econômico dos profissionais, mas esteve inversamente associado a idade dos mesmos. O mesmo também foi maior entre aqueles de cor da pela branca. Na análise ajustada, o conhecimento manteve-se associado as mesmas variáveis da análise bivariada, com o acréscimo da variável sexo, onde os homens apresentaram conhecimento superior (tabela 2). Profissionais de academias do sexo feminino e de cor da pele não branca apresentaram 0,6 e 2,0 pontos a menos, respectivamente, na média do escore de conhecimento quando comparados a seus grupos de referência. A idade dos professores esteve inversamente

Tabela 1. Descrição da população de profissionais segundo variáveis demográficas, social, econômica e nutricional em academias estratificadas por gênero.

\begin{tabular}{|c|c|c|c|}
\hline \multirow{2}{*}{ Variável } & Homens & Mulheres & Total \\
\hline & $\%$ & $\%$ & $\%$ \\
\hline \multicolumn{4}{|l|}{ Idade (anos) } \\
\hline $17-29$ & 56,3 & 67,5 & 61,0 \\
\hline $30-39$ & 32,3 & 22,0 & 28,0 \\
\hline $40-49$ & 7,6 & 6,7 & 7,2 \\
\hline $50-65$ & 3,8 & 3,8 & 3,8 \\
\hline \multicolumn{4}{|l|}{ Estado civil } \\
\hline Casado/Nive com companheiro & 31,6 & 26,3 & 29,4 \\
\hline Solteiro & 68,4 & 73,7 & 70,6 \\
\hline \multicolumn{4}{|l|}{ Cor de Pele } \\
\hline Branca & 83,3 & 92,3 & 87,1 \\
\hline Não branca & 16,7 & 7,7 & 12,9 \\
\hline \multicolumn{4}{|l|}{ Escolaridade } \\
\hline Ensino Fundamental Incompleto & 1,0 & 0,0 & 0,6 \\
\hline Ensino Fundamental Completo & 1,4 & 1,0 & 1,2 \\
\hline Ensino Médio Incompleto & 1,7 & 0,0 & 1,0 \\
\hline Ensino Médio Completo & 10,8 & 2,9 & 7,5 \\
\hline $\begin{array}{c}\text { Superior Incompleto em } \\
\text { Educação Física }\end{array}$ & 19,8 & 17,2 & 18,7 \\
\hline $\begin{array}{c}\text { Superior Completo em } \\
\text { Educação Física }\end{array}$ & 49,0 & 59,3 & 53,3 \\
\hline Superior Incompleto em outra área & 6,6 & 4,3 & 5,6 \\
\hline Superior Completo em outra área & 9,7 & 15,3 & 12,1 \\
\hline \multicolumn{4}{|l|}{ Nível econômico" ${ }^{\#}$} \\
\hline $1^{\circ}$ quartil de renda & 24,5 & 29,5 & 26,6 \\
\hline $2^{\circ}$ quartil de renda & 21,3 & 26,6 & 23,5 \\
\hline $3^{\circ}$ quartil de renda & 31,9 & 29,1 & 30,7 \\
\hline $4^{\circ}$ quartil de renda & 22,3 & 14,8 & 19,2 \\
\hline \multicolumn{4}{|l|}{ IMC $\left(\mathrm{kg} / \mathrm{m}^{2}\right)$} \\
\hline Eutrófico & 37,1 & 84,6 & 57,1 \\
\hline Sobrepeso & 53,1 & 12,0 & 35,8 \\
\hline Obesidade & 9,8 & 3,4 & 7,1 \\
\hline
\end{tabular}

associada ao escore de conhecimento e a variável nível econômico apresentou relação direta com o mesmo. Os profissionais com maior renda obtiveram 2,1 pontos a mais na média que os de menor renda.

A tabela 3 apresenta as 20 questões sobre conhecimento sobre AF e saúde estratificada por sexo e para toda amostra, com a frequência de respostas corretas em cada uma.

As atividades físicas básicas a serem indicadas, segundo a Organização Mundial da Saúde (OMS), estavam bem definidas para apenas 5,4\% dos profissionais atuantes nas academias. Setenta e seis porcento dos entrevistados afirmaram que a prática de exercício físico com o intuito de melhorar a aptidão cardiovascular é capaz de reduzir a frequência cardíaca de repouso em relação a mesma no início do programa de exercício físico.

Mais de $50 \%$ dos homens sabiam que o balanço energético positivo ocorre quando o consumo é maior do que o gasto calórico, contra $36,8 \%$ das mulheres. Quase 70\% dos profissionais não souberam informar o que os métodos de dobras cutâneas e o IMC estimam.

Em relação às estruturas musculares do manguito rotador e do quadríceps, 40,4\% e 37,8\% dos profissionais, respectivamente, souberam quais os músculos que as compõem. Segundo 67,5\% das mulheres, na fase excêntrica da ação muscular esquelética a origem proximal afasta-se da origem distal da musculatura. Em relação às recomendações de tempo semanal de AF de acordo com a OMS, pouco mais de 20\% dos profissionais souberam que adolescentes e adultos saudáveis devem realizar semanalmente, no mínimo, 300 e 150 minutos de AF, respectivamente.

A caminhada como fator de prevenção para a ocorrência de diabetes, de infarto do miocárdio e de acúmulo de gordura corporal foi reconhecida por mais de $89 \%$ dos profissionais que trabalhavam na área. Cerca de $72 \%$ dos profissionais responderam corretamente, afirmando que o treinamento resistido é indicado para prevenção e controle da perda de massa muscular com o envelhecimento (Tabela 3).

Tabela 2. Análise bruta e ajustada entre o escore de conhecimento sobre atividade física e saúde e as variáveis independentes.

\begin{tabular}{|c|c|c|c|c|}
\hline \multirow[b]{2}{*}{ Variável } & \multicolumn{2}{|c|}{ Análise Bivariada } & \multicolumn{2}{|c|}{ Análise ajustada } \\
\hline & $\begin{array}{c}\text { Conhecimento } \\
\text { (Média) }\end{array}$ & $\begin{array}{c}\text { Valor } \\
\mathrm{p}\end{array}$ & $\begin{array}{c}\text { Coeficiente } \beta \\
(\text { IC } 95 \%)\end{array}$ & $\begin{array}{c}\text { Valor } \\
p^{c}\end{array}$ \\
\hline Sexo & & $0,3^{a}$ & & 0,04 \\
\hline Masculino & 10,7 & & - & \\
\hline Feminino & 10,4 & & $-0,6(-1,2$ a $-0,1)$ & \\
\hline Idade (anos) & & $<0,001^{b}$ & & $<0,001$ \\
\hline $17-29$ & 11,1 & & - & \\
\hline $30-39$ & 10,2 & & $-0,9(-1,5$ a $-0,2)$ & \\
\hline $40-49$ & 9,8 & & $-1,4(-2,5$ a $-0,3)$ & \\
\hline $50-65$ & 8,1 & & $-3,1(-4,6 a-1,6)$ & \\
\hline Cor da pele & & $<0,001^{a}$ & & $<0,001$ \\
\hline Branca & 10,8 & & - & \\
\hline Não Branca & 9,0 & & $-2,0(-2,9 a-1,2)$ & \\
\hline Estado civil & & $0,3^{a}$ & & 0,6 \\
\hline Casado/Vive c/ comp. & 10,4 & & - & \\
\hline Solteiro & 10,7 & & $0,2(-0,5$ a 0,8$)$ & \\
\hline Escolaridade & & $<0,001^{b}$ & & $<0,001$ \\
\hline Ensino médio comp. & 7,3 & & - & \\
\hline $\begin{array}{c}\text { Superior completo/incompleto } \\
\text { em outra graduação }\end{array}$ & 8,4 & & $0,3(-0,8$ a 1,3$)$ & \\
\hline Estudante de EF & 10,9 & & $3,1(2,1$ a 4,1$)$ & \\
\hline Superior completo em EF & 11,9 & & $3,8(2,9$ a 4,7$)$ & \\
\hline Nível econômico" & & $0,003^{b}$ & & $<0,001$ \\
\hline $1^{\circ}$ quartil de renda & 10,0 & & - & \\
\hline $2^{\circ}$ quartil de renda & 10,5 & & $0,8(0,0$ a 1,6$)$ & \\
\hline $3^{\circ}$ quartil de renda & 11,1 & & $1,5(0,7$ a 2,3$)$ & \\
\hline $4^{\circ}$ quartil de renda & 11,2 & & $2,1(1,2$ a 3,0$)$ & \\
\hline $\mathrm{IMC}\left(\mathrm{kg} / \mathrm{m}^{2}\right)^{\&}$ & & $0,1^{b}$ & & 0,06 \\
\hline Eutrófico & 10,7 & & - & \\
\hline Sobrepeso & 10,6 & & $-0,4(-1,1$ a 0,3$)$ & \\
\hline Obesidade & 9,8 & & $-1,1(-2,2$ a 0,1$)$ & \\
\hline
\end{tabular}

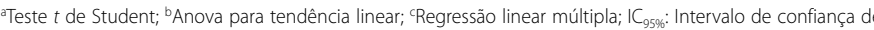
95\%."Nível econômico $n=485$; ${ }^{\circledR}$ MC (índice de massa corporal) n=494. EF: Educação Física. 
Tabela 3. Frequência de respostas corretas referente ao conhecimento sobre atividade física e saúde para toda amostra e estratificado para o sexo.

\begin{tabular}{|c|c|c|c|}
\hline \multirow{2}{*}{ Variável } & \multicolumn{3}{|c|}{ Frequência de respostas corretas (\%) } \\
\hline & Total & Homens & Mulheres \\
\hline $\begin{array}{l}\text { Segundo as últimas recomendações da Organização Mundial de Saúde², as atividades físicas básicas mais indicadas para indivíduos } \\
\text { adultos saudáveis são as de força muscular, aeróbias e de flexibilidade? }\end{array}$ & 5,4 & 4,9 & 6,2 \\
\hline $\begin{array}{l}\text { Quando alguém pratica exercício físico para melhorar a sua aptidão cardiovascular, a tendência é de que, após algum tempo, sua } \\
\text { frequência cardíaca (batimentos cardíacos) de repouso diminua em relação ao início do programa de exercício físico? }\end{array}$ & 76,3 & 77,4 & 74,6 \\
\hline $\begin{array}{l}\text { Em relação ao método de alongamento estático, está correto afirmar que ele é determinado pelo maior alcance voluntário, } \\
\text { utilizando-se da força dos músculos agonistas e o relaxamento dos músculos antagonistas? }\end{array}$ & 16,3 & 15,3 & 17,7 \\
\hline O balanço energético positivo ocorre quando o consumo é maior do que o gasto? & 44,67 & 50,35 & 36,8 \\
\hline $\begin{array}{l}\text { O método de composição corporal por dobras cutâneas e o IMC (Índice de Massa Corporal) indicam, respectivamente a estimativa } \\
\text { do percentual de gordura e o peso da gordura corporal? }\end{array}$ & 32,0 & 32,3 & 31,6 \\
\hline O manguito rotador é formado pelos músculos esqueléticos supraespinhoso, infraespinhoso, subescapular e redondo menor? & 40,4 & 38,1 & 43,1 \\
\hline $\begin{array}{l}\text { Ao realizarmos o exercício resistido de rosca direta, pode-se afirmar que, neste movimento de flexão de cotovelos, o músculo } \\
\text { bíceps braquial é antagonista? }\end{array}$ & 60,4 & 64,6 & 54,5 \\
\hline Em relação à fase excêntrica da ação muscular esquelética, é correto afirmar que a origem e inserção do músculo se afastam? & 64,0 & 61,5 & 67,5 \\
\hline $\begin{array}{l}\text { Os músculos esqueléticos que compõem o quadríceps são o vasto intermediário, o reto femoral, o tensor da fáscia lata e o } \\
\text { vasto lateral?. }\end{array}$ & 37,8 & 37,9 & 37,8 \\
\hline \multicolumn{4}{|l|}{ A caminhada, para um indivíduo previamente sedentário, pode modificar: } \\
\hline A pressão arterial de repouso & 59,0 & 62,0 & 55,5 \\
\hline A gordura corporal & 92,6 & 92,0 & 93,3 \\
\hline O risco de infarto do miocárdio & 90,3 & 89,6 & 91,4 \\
\hline O risco de contrair HIV & 99,0 & 99,0 & 99,0 \\
\hline O risco de diabetes & 89,1 & 88,9 & 89,5 \\
\hline A circulação sanguínea & 80,1 & 80,9 & 78,9 \\
\hline O nível do colesterol HDL & 37,2 & 42,0 & 30,6 \\
\hline $\begin{array}{l}\text { A duração semanal mínima em minutos de atividade física para que adultos e adolescentes saudáveis sejam considerados } \\
\text { ativos são, respectivamente, de: 1) } 120 \text { e } 250 \text { minutos/sem; 2) } 200 \text { e } 120 \text { minutos/sem; 3) } 150 \text { e } 250 \text { minutos/sem; 4) } 150 \text { e } \mathbf{3 0 0} \\
\text { minutos/sem; 5) } 300 \text { e } 200 \text { minutos/sem. }\end{array}$ & 21,3 & 21,9 & 20,6 \\
\hline $\begin{array}{l}\text { Mudanças que ocorrem a longo prazo em virtude do exercício físico são chamadas de: crônicas; agudas; contínuas; momentâneas } \\
\text { ou provisórias? }\end{array}$ & 32,0 & 33,3 & 30,1 \\
\hline $\begin{array}{l}\text { Para um adulto saudável, do sexo masculino, a medida máxima da circunferência da cintura em centímetros para ter risco baixo } \\
\text { de desenvolver algumas doenças é: 1) } 88 \mathrm{~cm} \text {; 2) } 90 \mathrm{~cm} \text {; 3) } 96 \mathrm{~cm} \text {; 4) } 102 \mathrm{~cm} \text {; 5) } 100 \mathrm{~cm} \text {. }\end{array}$ & 11,7 & 11,1 & 12,4 \\
\hline $\begin{array}{l}\text { Com o envelhecimento, ocorre diminuição da força e da massa muscular, as quais estão associadas a uma série de disfunções. Qual } \\
\text { atividade é mais indicada para prevenção e controle desse quadro: Aeróbia; Treinamento resistido; Hidroginástica; Alongamentos; Dança? }\end{array}$ & 72,6 & 72,6 & 72,7 \\
\hline
\end{tabular}

\section{DISCUSSÃO}

A média de idade dos profissionais de academias encontrada no presente estudo foi semelhante à de outros estudos ${ }^{14,15}$ e menor que a descrita em professores de educação física atuantes em escolas da cidade de Pelotas 9 . Dois estudos encontraram uma participação de $53,3 \%$ e $73,1 \%$ de homens trabalhando nesses locais ${ }^{16,17}$. Provavelmente, a predominância de profissionais do sexo masculino e com médias de idade mais baixa ocorreu devido ao grande número de academias que ofereciam a modalidade de musculação, atividade historicamente ligada aos homens, e pelo fato da maioria dos profissionais atuantes serem recém-formados. A maior parte dos entrevistados era solteiro $(70,6 \%)$, sendo que grande parte encontrava-se na faixa de idade entre 17 e 29 anos (72,4\%). Os dados encontrados são discordantes aos descritos pelo Instituto Brasileiro de Geografia e Estatística ${ }^{18}$, o qual mostra que as maiores taxas de nupcialidade no Brasil estão entre 25 e 29 anos (30,9\%) para homens e 20 e 24 (28,4\%) para mulheres. Em trabalhadores da mesma classe, o percentual de sujeitos casados também foi superior ao encontrado no presente estudo $(59,4 \%)^{19}$.

Encontrou-se uma prevalência elevada de profissionais de cor da pele branca atuantes nesses estabelecimentos (87,1\%). Estudos populacionais encontraram resultados semelhantes ao do presente estudo ${ }^{12,20}$. Em relação ao IMC dos profissionais de academias, um estudo com professores de EF de escolas desse mesmo município demonstrou índices semelhantes aos aqui encontrados ${ }^{9}$. Estudo populacional conduzido recentemente na cidade de Pelotas identificou prevalências de sobrepeso e obesidade mais elevadas que a encontrada nos professores ${ }^{21}$. Entretanto esse resultado deve ser analisado de forma cautelosa. 0 fato dos professores de academia estarem ligados à área da saúde e, especialmente, no presente município, mais de $80 \%$ deles trabalharem como professores de musculação e personal trainers, duas das modalidades de academia onde os profissionais apresentam considerável massa magra corporal, devem ter levado, equivocadamente, esses indivíduos a serem classificados com sobrepeso e obesidade pelo IMC.

Em relação à formação acadêmica, somente 53,3\% dos profissionais eram graduados em Educação Física. Prevalências maiores foram encontradas em outros estudos ${ }^{22,23}$ onde aproximadamente $64 \%$ dos profissionais eram formados. A alta prevalência de indivíduos leigos e estagiários trabalhando dentro das academias, possivelmente, gera impactos negativos na qualidade do atendimento prestado e na saúde dos clientes.

Apenas $21,5 \%$ dos profissionais acertaram mais de $70 \%$ das questões sobre AF e saúde, sendo que, somente 31,3\%, dos formados obtiveram este resultado. Cabral, Sousa e Raydan ${ }^{24}$, relataram que todos profissionais de academia entrevistados obtiveram $75,0 \%$ de acertos sobre conhecimento para prescrição de atividade física para a saúde. Já em relação aos conhecimentos sobre o sedentarismo como fator de risco para DANTs como diabetes tipo II, osteoporose e infarto do miocárdio, este percentual de acertos diminui para $62,5 \%$. A dificuldade das questões, além de temas mais específicos sobre AF e saúde abordados no instrumento e um menor percentual de profissionais graduados em Educação Física atuando nas academias de ginástica podem ter influenciado na baixa frequência de acerto do nosso estudo.

A AF é vista como um dos principais meios para se adquirir uma vida saudável e representa um importante componente do estilo de vida, contribuindo sobremaneira para a melhoria da qualidade de vida e do bem-estar ${ }^{25}$. É um fator de suma importância, tanto na 
prevenção, quanto no controle de certas DANTs como as doenças cardiovasculares, a obesidade e as dislipidemias ${ }^{26}$. A baixa prevalência de respostas corretas $(5,4 \%)$ referente à importância das atividades aeróbias, sobrecarga muscular e flexibilidade é preocupante visto que o estudo tratou majoritariamente com futuros profissionais e com professores graduados em Educação Física que atuavam em locais que apresentavam, como um dos principais objetivos, a melhoria da qualidade de saúde dos seus alunos.

Os profissionais, ao serem perguntados sobre a redução da pressão arterial de repouso, risco de diabetes e de infarto agudo do miocárdio, encontrou-se 59\%, 89,1\% e 90,3\%, respectivamente, que acreditavam que a caminhada ajudava na modificação desses parâmetros. Estudos discordam dos resultados encontrados em nosso trabalho, pois concluíram que há uma carência de conhecimento a respeito da prescrição de AF para pessoas com hipertensão arterial sistêmica e diabetes mellitus por profissionais de Educação Física ${ }^{15,16}$. Atividades como caminhar e correr tem sua prática facilitada por não exigir equipamentos caros ou locais específicos para sua execução ${ }^{13}$, podendo ser um importante fator de prevenção e controle destas doenças.

Os profissionais de academia apresentaram bom conhecimento (72,6\%) sobre a influência da prática de AF na diminuição da frequência cardíaca de repouso. Estudos têm examinado os efeitos benéficos da AF na massa e força muscular de idosos, demonstrando a manutenção dessa capacidade física ${ }^{29,30}$. Grande parte dos profissionais de academia reconheceu corretamente a relação entre atividades de treinamento resistido e o declínio de massa e força muscular nos idosos.

As variáveis sexo, idade, cor da pele, escolaridade e nível econômico apresentaram associação na análise ajustada com o escore de conhecimento. Os resultados vão ao encontro aos apresentados por Silveira e Silva ${ }^{13}$, que encontraram associação direta entre o conhecimento sobre AF com idade, nível econômico e escolaridade e com a cor da pele não branca.

Borges et al. ${ }^{11}$, verificaram que o nível socioeconômico e a escolaridade associaram-se diretamente ao conhecimento sobre sedentarismo, tabagismo e alimentação inadequada. Domingues et al. afirmam que

\section{REFERÊNCIAS}

1. Boutayeb A, Boutayeb S. The burden of noncommunicable diseases in developing countries. Int Equity Health. 2005;14(4)(1):2.

2. World Health Organization. Global recommendations on physical activity for health. Geneva:WHO 2010:8-10.

3. Bize R, Johnson JA, Plotnikoff RC. Physical activity level and health-related quality of life in the general adult population: a systematic review. Prev Med. 2007;45:401-15.

4. Stamatakis E, Ekelund U, Wareham NJ. Temporal trends in physical activity in England: The Health Survey for England 1991 to 2004. Prev Med. 2007;45(6):416-23.

5. DuBose KD, Kirtland KA, Hooker SP, Fields RM. Physical activity trends in South Carolina, 1994-2000. South Med J. 2004;97(9):806-10.

6. Brasil. Ministério da Saúde. Secretaria de Vigilância em Saúde. Vigitel Brasil 2011: Vigilância de Fatores de Risco e Proteção para Doenças Crônicas por Inquérito Telefônico. Ministério da Saúde, Secretaria de Vigilância em Saúde. Brasília: Ministério da Saúde; 2012.

7. Rojas PNC. Aderência aos programas de exercícios físicos em academias de ginástica na cidade de Curitiba-PR [Dissertação de mestrado], Florianópolis: Universidade Federal de Santa Catarina; 2003.

8. Silva MC, Rombaldi AJ, Azevedo MR, Hallal, PC. Participação atual e passada em academia de ginástica entre adultos: prevalência e fatores associados. Rev Bras Ativ Fis Saude. 2008;13:28-36.

9. Rombaldi AJ, Borges TT, Canabarro LK, Corrêa LQ, Neutzling MB. Conhecimento de professores de educação física sobre fatores de risco para doenças crônicas de uma cidade do sul do Brasil. Rev Bras Cineantropom Desempenho Hum. 2012;14:61-72.

10. Hartwig TW, Silva MC, Reichert FF, Rombaldi AJ. Condições de saúde de trabalhadores de academias da cidade de Pelotas-RS: um estudo de base populacional. Rev Bras Ativ Fis Saúde. 2013;17:500-11.

11. Borges TT, Rombaldi AJ, Knuth AG, Hallal PC. Conhecimento sobre fatores de risco para doenças crônicas: estudo de base populacional. Cad Saúde Pública. 2009;25:1511-20.

12. Domingues MR, Araújo CL, Gigante DP. Conhecimento e percepção sobre exercício físico em uma população adulta urbana do sul do Brasil. Cad Saúde Pública. 2004;20:204-15.

13. Silveira EF, Silva MC. Conhecimento sobre atividade física dos estudantes de uma cidade do sul do Brasil. Motriz. 2011;17:456-67.

14. Mendes, AD. Atuação profissional e condições de trabalho do educador físico em academias de atividades físicas. [Dissertação de Mestrado], Brasília: Universidade de Brasília; 2010.

15. Monteiro LZ, Spinato IL, Silva CABd, Santos ZMdSA, Montenegro Júnior RM. Nível de conhecimento do profissional de educação física frente a alunos com hipertensão arterial nas academias de ginástica. Revista Bras Cineantropom Desempenho Hum. 2010;12:262-8. quanto maior a escolaridade, maior conhecimento dos indivíduos, fato esse sustentado pela associação linear positiva encontrada em seu estudo. A associação entre conhecimento e nível econômico encontrada no presente estudo pode demonstrar que grupos menos favorecidos financeiramente podem possuir menor acesso a fontes de atualização e de informação ou, por receberem menos, dedicarem-se com menor afinco à busca de novos conhecimentos. No entanto, já era esperado que os profissionais formados em Educação Física apresentassem um conhecimento mais elevado que os nãos formados.

O ineditismo em avaliar de forma censitária o conhecimento sobre AF e saúde de profissionais de academias de uma cidade de porte médio do Rio Grande do Sul, o baixo índice de perdas e recusas, e a logística do estudo são pontos importantes a serem destacados. Entretanto, a inexistência de um instrumento padrão-ouro para avaliar a medida do conhecimento sobre AF e saúde dos profissionais das academias, é fator que deve ser lembrado.

\section{CONCLUSÕES}

O escore de conhecimento sobre AF e saúde foi baixo entre os profissionais que atuavam em academias de ginástica da cidade de Pelotas. Além disso, ser do sexo masculino, faixa etária jovem, de cor da pele branca, de maior nível econômico e maior escolaridade, estiveram associados a maiores escores de conhecimento. Tal resultado torna-se preocupante frente ao número de pessoas atendidas por esses profissionais, os quais podem não estar ajudando na melhoria das condições de saúde desses indivíduos ou, em uma visão mais pessimista, piorando esta condição. Sugere-se uma maior fiscalização para a atuação dos profissionais nesse mercado, visto que quase $45 \%$ dos ministrantes de aulas em academias atuam ilegalmente nestes espaços de promoção de AF. Além disso, enfatizamos a necessidade de novos estudos nessa população em outras cidades do Brasil de forma a reforçar o ainda recente conhecimento sobre o tema.

Todos os autores declararam não haver qualquer potencial conflito de interesses referente a este artigo.

16. Monteiro LZ, Spinato IL, Silva CAB, Pinheiro MHNP, Santos ZMSA, Júnior RMM. Conhecimento do profissional de educação física frente à atuação com portadores de diabetes mellitus nas academias de ginástica de Fortaleza, CE. Rev Bras Educ Fís Esporte. 2009;23:135-42.

17. Palma A, Assis M. Uso de esteroides anabólico androgênicos e aceleradores metabólicos entre professores de educação física que atuam em academias de ginástica. Rev Bras Cien Esporte. 2008;27:75-92.

18. Instituto Brasileiro de Geografia e Estatística. Estatística do Registro Civil. 2009;36:1-186.

19. Silva JVP, Nunez PRM. Qualidade de vida, perfil demográfico e profissional de professores de Educação Física. Pensar Prát. 2009;12:1-12.

20. Mendes MA, Rombaldi AJ, Azevedo MR, Bielemann RM, Hallal PC. Fontes de informação sobre a importância da atividade física: estudo de base populacional. Rev Bras Ativ Fis Saude. 2012;15:163-9.

21. Linhares RS, Horta BL, Gigante DP, Dias-da-Costa JS, Olinto MTA. Distribuição de obesidade geral e abdominal em adultos de uma cidade no Sul do Brasil. Cad Saúde Pública. 2012;28:438-47.

22. Antunes AC. Perfil profissional de instrutores de academias de ginástica e musculação. Lec Educ Fis Dep 2003;9(60).

23. Marcellino, NC. Academias de ginástica como opção de lazer. Rev Bras Ciên Mov. 2003;11:49-54.

24. Cabral IA, Sousa MAM, Raydan FPS. Análise do conhecimento dos profissionais de educação física em relação à atividade física como promotora da saúde. Movimentum - Rev Dig Educ Fís. 2007;2:1-11.

25. Nahas MV. Atividade física, saúde e qualidade de vida: conceitos e sugestões para um estilo de vida ativo. $4^{\mathrm{a}}$ ed. Londrina: Midiograf, 2006.

26. Guedes DP, Guedes J, Barbosa DS, Oliveira J. Níveis de prática de atividade física habitual em adolescentes. Rev Bras Med Esporte. 2001;7:187-99.

27. Vatten $\sqcup$, Nilsen TI, Holmen J. Combined effect of blood pressure and physical activity on cardiovascular mortality. J Hypertens. 2006;24:1939-46.

28. Sato KK, Hayashi T, Kambe H, Nakamura Y, Harita N, Endo G, et al. Walking to work is an independent predictor of incidence of type 2 diabetes in japanese men the kansai healthcare study. Diabetes Care. 2007;30:2296-8.

29. Hughes VA, Frontera WR, Wood M, Evans WJ, Dallal GE, Roubenoff R, et al. Longitudinal muscle strength changes in older adults: influence of muscle mass, physical activity, and health. J Gerontol A Biol Sci Med Sci. 2001;56:209-17.

30. Goodpaster BH, Chomentowski P, Ward BK, Rossi A, Glynn NW, Delmonico MJ, et al. Effects of physical activity on strength and skeletal muscle fat infiltration in older adults: a randomized controlled trial. J Appl Physiol. 2008;105:1498-503. 